\title{
Correlative SPM/TEM Investigation of the Electrochemical Deposition of Lithium Metal
}

Colin Campbell ${ }^{1,2}$, Byeongdu Lee ${ }^{3}$, Yong Min Lee ${ }^{4}$, Kuk Young Cho ${ }^{5}$, Young-Gi Lee ${ }^{6}$, Seungbum Hong $^{7}$, Charudatta Phatak

1. Materials Science Division, Argonne National Laboratory, Lemont, USA.

2. Department of Materials Science \& Engineering, Northwestern University, Evanston, USA.

3. X-ray Sciences Division, Argonne National Laboratory, Lemont, USA.

4. Department of Energy Science and Engineering, Daegu Gyeongbuk Institute of Science and Technology (DGIST), Daegu, Republic of Korea.

${ }^{5 .}$ Deparment of Materials Science and Chemical Engineering, Hanyang University, Ansan, Republic of Korea.

${ }^{6}$ ICT Materials and Components Research Laboratory, ETRI, Republic of Korea.

7. Dept. Mater. Sci. \& Eng., KAIST, Daejeon, Republic of Korea.

There is considerable interest in high-capacity battery technology, for a variety of reasons including improved lifetime of electronic devices and applications in long-range electric vehicles; due to their excellent diffusion properties and significant cell voltage, lithium-ion batteries are common choices where high specific capacity or capacity by weight is required. Much of the effort to improve these devices has been spent to improve the cathode material for these batteries, but most commercial Li-ion batteries still use a graphite anode. Compared to a graphite anode, lithium has more than 10x the theoretical specific capacity and a lower reduction potential [1], implying a significant increase in power density is possible were $\mathrm{Li}$ anodes to be implemented, but they are prone to failure involving internal shorts or capacity depletion as a result of conductive metal structures called dendrites. In the interest of more completely understanding the conditions of dendrite formation, in-situ measurements of lithium anodes and dendrites have been performed. In this work, we intend to use various techniques of scanning probe microscopy [2] in parallel with TEM methods to examine the formation of lithium dendrites in patterned electrodes under various conditions.

In-situ observation of the deposition of $\mathrm{Li}$ on a gold substrate were performed using an in-situ liquid cell TEM, in a method similar to that performed by Zhiyuan Zeng [3]. We were able to observe a solid crystalline deposition by decomposition of the electrolyte near the working electrode after cyclic voltammetry as shown in Figure 1. Future experiments will include a supply of lithiated NMC attached to one electrode to serve as a source of Li without the decomposition of electrolyte salts, and a carbon electrode with significantly sharp edges to permit the investigation of Li intercalation, deposition, and SEI formation in the vicinity of curved surfaces. As the previous work suggests that patterned electrodes supply current inhomogeneously [4], this inhomogeneous current density could also mean that the SEI that forms near regions of high curvature is different than that which forms in flatter areas, which we can verify on the edges of the carbon electrode we intend to use.

Snapshots of SEI formation have been observed with a sealed electrochemistry cell for AFM. The Li sample was cycled with a square wave voltage profile, using EC/EMC/LiPF6 electrolyte and a graphite counter electrode, and was purged without exposure to air, after cycling, in an argon glove box. Topographic AC-AFM scans before and after cycling are shown in Figure 2 which indicated formation of a soft SEI layer. Further experiments will include Kelvin probe force microscopy to investigate the 
variation of surface potential of the SEI in the vicinity of a scratch made in the metal to understand the effect of patterning.

In conclusion, we will discuss the results obtained from correlative microscopy to observe the change of morphology of Li deposited on electrode in an in-situ electrochemical environment [5].

\section{References:}

[1] X B Cheng et al, Chemical Reviews 117 (2017), p. 10403.

[2] C. Campbell et al, Scientific Reports 8 (2018), p. 2514.

[3] Z Zheng et al, Nano Letters 14 (2014), p. 1745

[4] M S Park et al, Scientific Reports 4 (2014), p. 3815.

[5] This work was supported by the International Collaborative Energy Technology R\&D Program of the Korea Institute of Energy Technology Evaluation and Planning (KETEP), granted financial resource from the Ministry of Trade, Industry \& Energy, Republic of Korea. (No. 20158510050020).

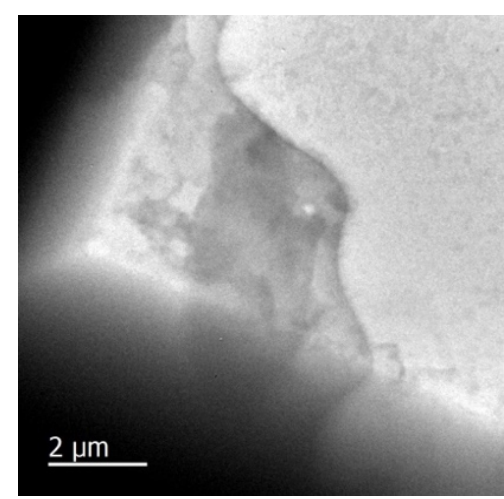

Figure 1. Micrographs of solid depositing on the Au working electrode of the TEM fluid cell after cyclic voltammetry was performed using EC/EMC/1M LiPF6 electrolyte.
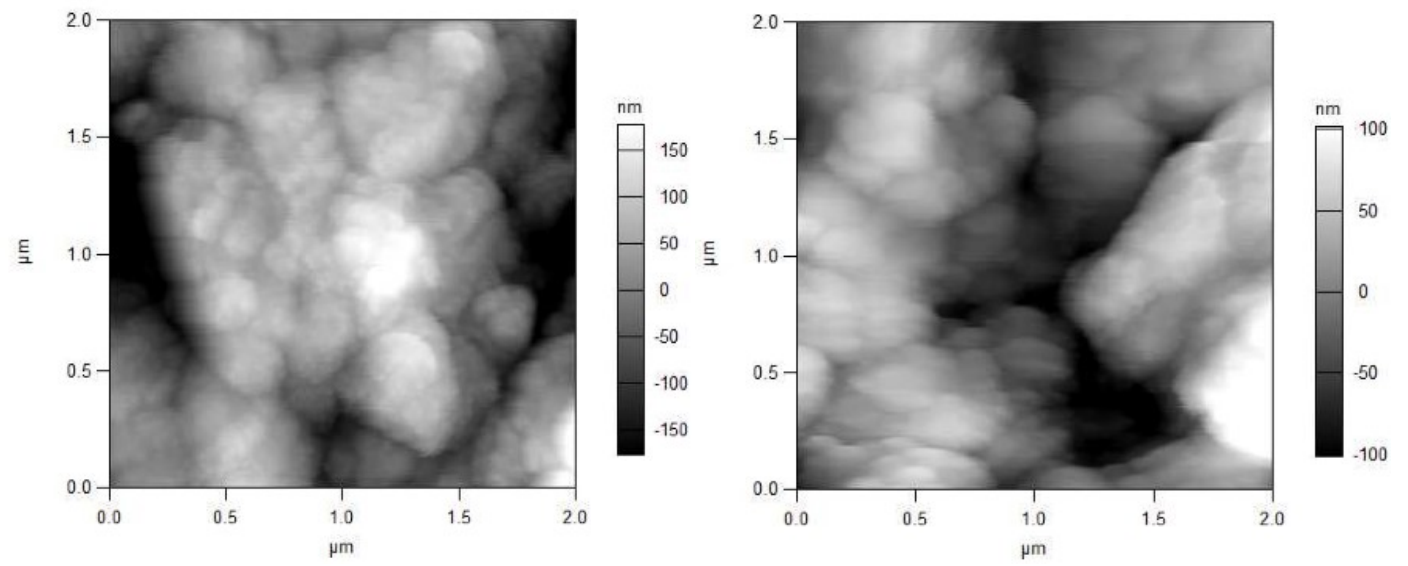

Figure 2. Height profiles of a lithium anode after being exposed to EC/EMC/1M LiPF6 electrolyte before (left) and after (right) cycling with a square wave profile that primarily stripped Li. No change in feature size is noted, although a difference in average height variation is apparent. As these are not the same area, more experiments are required to determine the statistical significance of these differences. 